\title{
Intervención breve para adolescentes que inician el consumo abusivo de alcohol y otras drogas: su eficacia en un escenario clínico
}

\author{
Carlos Augusto Rodríguez Durán', Leticia Echeverría San Vicente', Kalina Isela Martínez Martínez², \\ Silvia Morales Chainél \\ ' Facultad de Psicología, Universidad Nacional Autónoma de México, México \\ 2 Universidad Autónoma de Aguascalientes, Centro de Ciencias Sociales y Humanidades, Departamento de Psicología, Aguascalientes, México
}

\section{RESUMEN}

Introducción: en las últimas décadas, se ha observado en los adolescentes mexicanos un incremento en la cantidad y frecuencia del consumo de alcohol y drogas ilícitas; los programas de intervención breve han demostrado ser eficaces para reducir el consumo y abuso de sustancias en diversas poblaciones. Objetivo: determinar la eficacia del Programa de Intervención Breve para Adolescentes que Inician el Consumo Abusivo de Alcohol y otras Drogas (PIBA), así como el mantenimiento del cambio en los jóvenes que asistieron. Método: se utilizó un diseño de medidas repetidas, no probabilístico y sin grupo control. En el seguimiento participaron 60 usuarios consumidores de sustancias de entre 16 y 20 años de edad, de ambos sexos, habitantes de la Ciudad de México. Resultados: al comparar la preintervención vs. el seguimiento, se encontró que los adolescentes que participaron en el PIBA mantuvieron una reducción en su patrón de consumo (cantidad, frecuencia y tiempo de abstinencia) de alcohol, mariguana e inhalables; disminuyeron los problemas asociados con el consumo; e incrementaron su nivel de autoeficacia percibida. La mejoría fue mayor en quienes concluyeron la intervención breve que en quienes no terminaron todas las sesiones. Conclusiones: el PIBA fue coadyuvante en la consecución de las metas propuestas por la mayoría de los adolescentes que asistieron a este tratamiento psicológico.

Palabras clave: adolescentes, consumo de sustancias, intervenciones breves.

\begin{abstract}
SUMMARY
Introduction: in recent decades, it has been observed an increase in the amount and frequency of alcohol intake and consume of illicit drugs in Mexican adolescents. Brief intervention programs have been effective reducing consumption and substance abuse, in diverse teens populations. Objective: to determine the effectiveness of the Brief Intervention Program for Adolescents Initiating Abusive Consumption of Alcohol and Other Drugs (PIBA), as well as, the maintenance of the change in the youngsters who were attended. Method: a repeated measures design, pre and post test, no probabilistic and without control group, was used. In the follow-up, 60 substance users participated, between 16 and 20 years of age, of both sexes, inhabitants of the Mexico City. Results: by comparing the pre-intervention versus follow-up, teens who participated in the PIBA maintained a reduction in their consumption pattern (amount, frequency and time of abstinence substances alcohol, marijuana and inhalants), decreased the problems associated with consumption, and they increased their level of perceived self-efficacy. The improvement was greater in the group that concluded the brief intervention than in the group that left it unfinished. Discussion: PIBA was an important aid in achieving the goals proposed by adolescents who attended this psychological treatment.
\end{abstract}

Keywords: adolescents, substance use, brief interventions.

\footnotetext{
Autor de correspondencia:

Carlos Augusto Rodríguez Durán. Retorno 17 núm. 35, col. Avante, del. Coyoacán. C.P. 04460, Ciudad de México.

Correo electrónico: carlosreduran@hotmail.com

Recibido: 14 de noviembre de 2016

Aceptado: 20 de abril de 2017

DOI: $10.28931 /$ riiad.2017.1.03
} 


\section{INTRODUCCIÓN}

La adolescencia es una etapa de vida crucial para toda persona, es un periodo crítico y definitorio en el desarrollo humano; en ella existen cambios a nivel físico, mental y social que se deben desarrollar de una forma adecuada para poder alcanzar la madurez necesaria en la etapa adulta (Mingote \& Requena, 2008; Peñaloza, 2010).

De acuerdo con la Encuesta Nacional de Consumo de Drogas en Estudiantes (2014), en los últimos años se ha observado en los adolescentes mexicanos un incremento en la cantidad y la frecuencia del consumo de alcohol y drogas ilícitas, en específico de mariguana e inhalables; de manera paralela, la edad de inicio del consumo ha ido disminuyendo (Instituto Nacional de Psiquiatría Ramón de la Fuente Muñiz, Comisión Nacional contra las Adicciones, \& Secretaría de Salud, 2015).

El uso de sustancias psicoactivas modifica las capacidades físicas, mentales y conductuales de los jóvenes. Cada droga causa reacciones específicas en la psique y el organismo; los consumidores la buscan por sus efectos placenteros o para disminuir sentimientos displacenteros. Sin embargo, su abuso conlleva un potencial de daños a corto y largo plazo, así como consecuencias negativas psicológicas y sociales en la vida de los consumidores y sus familias (Tiburcio, Carreño, Martínez, Echeverría, \& Ruiz, 2009).

Las intervenciones breves son terapias psicológicas cognitivo-conductuales, de corta duración y alta efectividad, de costos reducidos y resultados favorables en la práctica clínica (Barragán, González, Medina-Mora, \& Ayala, 2005; Hewitt \& Gantiva, 2009). El enfoque cognitivo-conductual considera que las conductas adictivas son hábitos sobreaprendidos que pueden ser modificados mediante técnicas de autocontrol (Hervás \& Tomás-Gradolli, 2002; Tiburcio, Carreño, Martínez, Echeverría, \& Ruiz, 2009).

En los ámbitos internacional y nacional se ha demostrado que las intervenciones breves basadas en la teoría del aprendizaje social, que además utilizan componentes de la entrevista motivacional, la prevención de recaídas y las técnicas de autocontrol son efectivas para personas con un patrón de consumo problemático que aún no han desarrollado dependencia (Ayala, 2001; Ayala, Echeverría, Sobell, \& Sobell, 1998; Babor \& Grant, 1992; Espadero, 2008; Heather \& Kaner, 2001; Hewitt \& Gantiva, 2009; Martínez, 2003; Sobell \& Sobell, 1996; Tiburcio et al., 2009).

En México, Martínez y colaboradores (Martínez, 2003; Martínez, Ávila, Pacheco, \& Lira, 2011; Martínez \& Cabrera, 2011; Martínez, Pedroza, Salazar, \& Vacio, 2010; Martínez, Salazar, Jiménez, Ruiz, \& Ayala, 2012; Martínez, Salazar, Pedroza, Ruiz, \& Ayala, 2008) han publicado diversos artículos en los que se muestran los resultados del Programa de Intervención Breve para Adolescentes que Inician el Consumo de Alcohol y otras Drogas (PIBA). El PIBA es una intervención cognitivo-conductual que utiliza la entrevista motivacional, técnicas de autocontrol y prevención de recaídas para la reducción del consumo de sustancias y problemas asociados.

Las mediciones realizadas en la línea base, la intervención y los seguimientos del PIBA muestran un aumento en la autoeficacia percibida, una reducción en el patrón de consumo de los jóvenes, tanto en cantidad como en frecuencia, y una disminución de los accidentes y problemas relacionados con la forma de consumo (familiares, escolares, económicos, de salud, emocionales y sociales).

La autoeficacia tiene un papel relevante en el inicio de la conducta de consumo, el curso del tratamiento, el mantenimiento de la abstinencia y la prevención de recaídas. Las expectativas de autoeficacia se refieren a las creencias del individuo acerca de su habilidad para afrontar de manera exitosa alguna situación; están establecidas, parcialmente, por el repertorio de habilidades de afrontamiento del individuo y por la valoración de la efectividad relativa, en relación con las demandas específicas de la situación (Bandura, 1986). Determinan que la conducta de afrontamiento se inicie o no, la cantidad de esfuerzo necesario para ejercerla y cuándo se tendrá que continuar un intento de afrontamiento de cara a los obstáculos y experiencias aversivas.

La autoeficacia influye sobre la conducta del individuo, a través de los sistemas motivacional, cognitivo y emocional. Si una persona tiene poca autoeficacia, debido a la carencia de habilidades necesarias, es de esperarse que presente pensamientos negativos o distorsionados, así como una motivación reducida para tratar de afrontar las situaciones (Bandura, 1994).

Por último, es importante señalar que con el término "seguimiento", se hace alusión al contacto individual, sistemático y estructurado posterior a uno o más intervalos de tiempo asignados después del tratamiento. Los seguimientos son una fuente confiable que nos ayuda a documentar resultados sobre el mantenimiento del cambio, son útiles para diseñar y evaluar futuras intervenciones y son una buena inversión en términos de costo-efectividad (considerando tiempo y esfuerzo en el tratamiento). Además, generan impacto en los usuarios, ya que a través de ellos se detectan recaídas y, a partir de la retroalimentación obtenida después del tratamiento, se puede determinar el tipo de apoyo que requiere la persona (Hester \& Miller, 1989).

Conocer resultados y conclusiones derivados de los seguimientos puede contribuir a la planeación y la evaluación de tratamientos y técnicas efectivas de in- 
tervención, así como al mejoramiento de sus principales componentes (Martínez, Ávila et al., 2011). Sin embargo, existen escasos estudios que reporten seguimientos realizados después de un año o más tiempo de concluida la intervención breve. Por lo antes expuesto, el objetivo de este estudio fue determinar la eficacia de una intervención breve dirigida a población adolescente que se inicia en el consumo abusivo de alcohol y otras drogas, a través de la evaluación del mantenimiento del cambio, realizada mediante un seguimiento.

Se efectuaron evaluaciones preintervención y un seguimiento en cada participante. Las variables utilizadas para determinar la eficacia del programa fueron: cantidad de consumo, frecuencia de consumo por semana, consecuencias negativas asociadas con el consumo y autoeficacia percibida para abandonar el consumo de sustancias.

Un aspecto novedoso del presente estudio fue que permitió corroborar los resultados positivos del PIBA en un escenario de formación de terapeutas a nivel de maestría: el Centro de Prevención en Adicciones de la Facultad de Psicología de la UNAM, cuya misión es promover la formación profesional del estudiante y, de esta forma, impactar en el campo de la prevención del consumo de drogas.

La hipótesis propuesta fue que los adolescentes que concluyeron la intervención breve mantendrían mayores cambios positivos en las variables dependientes evaluadas, en contraste con los usuarios que desertaron de la intervención breve.

\section{MÉTODO}

\section{Participantes}

La selección de la muestra fue intencional y no probabilística, se trabajó con 60 adolescentes que asistieron al Centro de Prevención en Adicciones de la Facultad de Psicología de la Universidad Nacional Autónoma de México (UNAM), participaron en el PIBA y cumplieron con los siguientes requisitos:

a) Ser adolescentes usuarios de alcohol, con consumo mayor a cuatro unidades de bebida estándar por ocasión-clasificado como consumo de riesgo-, pero que no han manifestado indicadores de dependencia.

b) Ser adolescentes que se están iniciando en el consumo de sustancias ilegales como mariguana o inhalables, pero que no han manifestado indicadores de dependencia de la droga.

c) Reportar consumo de riesgo y problemas asociados con el consumo de alcohol o drogas ilegales, pero sin presentar indicadores de dependencia.
La muestra a la que se le hizo el seguimiento quedó conformada por un total de 60 adolescentes habitantes de la Ciudad de México, de entre 16 y 20 años de edad (cabe señalar que al momento de la intervención, todos los usuarios eran menores de edad; sin embargo, durante el seguimiento, algunos cumplieron 20 años), que asistieron entre 2010 y 2014 a recibir tratamiento al Centro de Prevención en Adicciones Dr. Héctor Ayala Velázquez (CPAHAV). 30 de estos participantes (50\%) concluyeron la intervención breve (grupo alta), mientras que los 30 restantes (50\%) dejaron inconclusa la intervención (grupo baja).

En las Tablas 1, 2 y 3 se señalan las características sociodemográficas de la muestra, la sustancia psicoactiva consumida y el tiempo que pasó antes de realizar el último seguimiento, mismo que permitió la evaluación de la muestra en este estudio.

\section{Instrumentos, técnicas de medición}

A) Instrumentos de medición preintervención:

\section{Entrevista inicial}

Desarrollada por Saucedo y Salazar en 2004 (citado en Martínez et al., 2012), la entrevista inicial es un instrumento que permite obtener información sobre diversos indicadores, como patrón de consumo de sustancias, historia de consumo, disposición al cambio, situación social y familiar, administración del tiempo libre, sexualidad, situación laboral, salud física y consecuencias adversas asociadas con el uso de sustancias.

II. Cuestionario de Tamizaje de los Problemas en los Adolescentes (POSIT)

Desarrollado por los autores Mariño, González-Forteza, Andrade y Medina-Mora en 1997, el POSIT consta de 81 reactivos que contemplan siete áreas de funcionamiento; para este programa sólo se utilizaron los 17 reactivos del área uso/abuso de sustancias. La confiabilidad interna del instrumento es de .90 , obtenida a través del $a$ de Cronbach. La finalidad de su aplicación es la identificación de adolescentes que presentan al menos una consecuencia negativa asociada con el consumo de sustancias psicoactivas (Martínez et al., 2008).

B) Instrumentos de medición aplicados en el seguimiento:

\section{Línea Base Retrospectiva (LIBARE)}

Desarrollada por Sobell y Sobell en 1979, la LIBARE se utiliza para medir el patrón de consumo de sustancias 
Tabla 1

Características sociodemográficas de la muestra

$\begin{array}{cc}\text { Grupo alta } & \text { Grupo baja } \\ (n=30) & (n=30) \\ n(\%) \circ M(D E) & n(\%) \circ M(D E)\end{array}$

\begin{tabular}{lcc}
\hline Sexo & & $24(80 \%)$ \\
Hombres & $20(66.6 \%)$ & $6(20 \%)$ \\
Mujeres & $10(33.3 \%)$ & $18.4(1.3)$ \\
Edad (en años) & $18.1(1.5)$ & $30(100 \%)$ \\
Estado civil & & $0(0 \%)$ \\
Solteros & $29(96.66 \%)$ & $0(0 \%)$ \\
Unión libre & $1(3.3 \%)$ & $5(16.6 \%)$ \\
Escolaridad & & $21(70 \%)$ \\
Primaria & $1(3.3 \%)$ & $3(10 \%)$ \\
Secundaria & $1(3.3 \%)$ & $1(3.3 \%)$ \\
Bachillerato trunco & $20(66.6 \%)$ & $17(56.6 \%)$ \\
Bachillerato completo & $2(6.6 \%)$ & $6(20 \%)$ \\
Licenciatura en proceso & $6(20 \%)$ & $1(3.3 \%)$ \\
Ocupación & & $2(6.6 \%)$ \\
Estudiantes & $20(66.6 \%)$ & $2(6.6 \%)$ \\
Trabajo tiempo completo & $6(20 \%)$ & $2(6.6 \%)$ \\
Trabajo medio tiempo & $2(6.6 \%)$ & $2(6.6 \%)$ \\
Trabajo temporal & $0(0 \%)$ & $0(0 \%)$ \\
Estudian y trabajan & & \\
Sin ocupación & & \\
\hline
\end{tabular}

Tabla 2

Sustancia consumida por los integrantes de la muestra

\begin{tabular}{lcc}
\hline & $\begin{array}{c}\text { Grupo alta } \\
(n=30) \\
n(\%)\end{array}$ & $\begin{array}{c}\text { Grupo baja } \\
(n=30) \\
n(\%)\end{array}$ \\
\hline $\begin{array}{l}\text { Sexo } \\
\text { Hombres }\end{array}$ & $20(66.6 \%)$ & $24(80 \%)$ \\
Mujeres & $10(33.3 \%)$ & $6(20 \%)$ \\
Sustancia & & $7(23.3 \%)$ \\
Alcohol & $6(20 \%)$ & $19(63.3 \%)$ \\
Mariguana & $18(60 \%)$ & $4(13.3 \%)$ \\
\hline Inhalables & $6(20 \%)$ & \\
\hline
\end{tabular}

(cantidad y frecuencia) antes, durante y después de un tratamiento; con ella se miden tres meses de consumo al inicio del tratamiento. Tiene una confiabilidad test-retest de .91 y la correlación del consumo entre el informe del individuo y el del colateral es de .82 (Echeverría, Ruiz, Salazar, Tiburcio, \& Ayala, 2004).
II. Cuestionario de Confianza Situacional forma breve (CCS)

Este instrumento fue desarrollado por Annis y Davis (1988), quienes se basaron en el concepto de autoeficacia de Bandura. A los usuarios se les pide que indi- 
Tabla 3

Tiempo que pasó entre la última visita al tratamiento y el seguimiento

\begin{tabular}{lcc}
\hline & $\begin{array}{c}\text { Grupo alta } \\
(n=30) \\
n(\%)\end{array}$ & $\begin{array}{c}\text { Grupo baja } \\
(n=30) \\
n(\%)\end{array}$ \\
\hline Tiempo & & $0(0 \%)$ \\
Seis meses & $2(6.6 \%)$ & $6(20 \%)$ \\
Un año & $7(23 \%)$ & $14(46.6 \%)$ \\
Dos años & $11(36.6 \%)$ & $10(33.3 \%)$ \\
Tres años & $9(30 \%)$ & $0(0 \%)$ \\
Cuatro años & $1(3.3 \%)$ & 0 \\
\hline
\end{tabular}

quen, en una escala de 0 a 100\%, qué tan confiados o seguros se sienten en el momento presente para resistir la tentación de consumir alguna droga en la situación imaginaria que se les plantea. Se utilizó la versión breve del Cuestionario de Confianza Situacional que consta de ocho reactivos (Echeverría \& Ayala, 1997); en esta versión adaptada a la población mexicana, la validez del constructo fue adecuada al correlacionar cada categoría con medidas de consumo, lo que permitió predecir situaciones de recaídas.

\section{Entrevista de seguimiento}

Este instrumento tiene el propósito de identificar los avances del adolescente en la consecución de su meta, después de concluida la intervención. La entrevista de seguimiento que fue utilizada para esta investigación se desarrolló en el CPAHAV, con base en los indicadores de eficacia de las intervenciones breves (Echeverría \& Ayala, 1997), e incluye las principales características que se deben evaluar en un seguimiento (Hester \& Miller, 1989). Para esta investigación se midieron las siguientes dimensiones: patrón de consumo y consecuencias adversas asociadas con el consumo.

C) Instrumentos de medición aplicados en el seguimiento:

\section{Entrevista colateral}

Este instrumento es complementario a la entrevista de seguimiento. Es útil para contrastar la información proporcionada por el adolescente con lo reportado por alguno de sus familiares; indaga la cantidad y la frecuencia del consumo de sustancias del usuario, así como su parentesco y la frecuencia de reunión del adolescente con sus colaterales.

\section{Consentimiento informado}

El consentimiento informado es un documento que el usuario firma si está de acuerdo con participar en la investigación. Explica que el seguimiento tiene la intención de apoyar el proceso de cambio posterior a la intervención y en él se da a conocer la confidencialidad de los datos que el usuario reporta.

\section{Diseño de investigación}

Se utilizó un diseño intra-sujetos de medidas repetidas en 30 usuarios con alta y 30 usuarios dados de baja del programa de intervención. Se aplicó una evaluación previa y posterior al estímulo o intervención breve, a fin de medir el cambio en las variables dependientes. La muestra se obtuvo por conveniencia y de manera no aleatoria.

\section{Consideraciones éticas}

En este estudio, las actividades para la recolección de los datos cumplieron con los lineamientos que marcan tanto el Código Ético del Psicólogo como la American Psychological Association. Los participantes firmaron un formato de consentimiento informado en el que se describen las características de la intervención, se señala el carácter confidencial de la información y se les da a los firmantes la libertad de abandonar el tratamiento en el momento que lo deseen, sin que esto tenga repercusión en sus derechos y obligaciones; los participantes, por su parte, otorgan a quienes realizan el estudio la posibilidad de utilizar los datos recabados con fines de investigación. En el caso de que los participantes fueran menores de edad, los padres o tutores firmaron, también, el formato de consentimiento.

\section{Procedimiento}

El PIBA está diseñado para aplicarse en cinco etapas: 
1. La admisión, en la que se determina si los adolescentes son candidatos para recibir la intervención.

2. La evaluación, con la que se hace una valoración de la historia de consumo y su patrón en los últimos seis meses; sirve también para identificar las principales situaciones de riesgo que llevan al usuario a consumir, así como su nivel de autoeficacia percibida y su idea sobre la severidad del consumo.

3. La inducción al tratamiento, con la que se motiva al adolescente a iniciar un cambio en su conducta de consumo para que permanezca y se adhiera al tratamiento; en esta etapa se utilizan técnicas de entrevista motivacional.

4. La intervención, dividida en varias sesiones. En la primera se hace un balance entre las ventajas y desventajas del consumo de sustancias psicoactivas, se enumeran las razones que tiene el participante para cambiar y se establece una meta de tratamiento. En la segunda, se identifican las situaciones de riesgo y se desarrollan, junto con el participante, estrategias de cambio o planes de acción. En la tercera, se identifican las metas que el participante quiere alcanzar a corto y largo plazo y se analiza la forma en la que el consumo influye sobre ellas. En la cuarta, se retroalimenta al participante sobre los cambios logrados, se establece una segunda meta de consumo y se planea cómo se va a mantener una vez terminado el tratamiento. Estas sesiones se realizan una vez por semana en formato individual; y los seguimientos, a los tres y seis meses de haber concluido la intervención (Martínez et al., 2010).

A los usuarios que llegaron por primera vez al CPAHAV, se les hizo una valoración inicial; quienes cumplieron con los requisitos de ingreso al PIBA iniciaron la intervención. Aquellos que no fueron candidatos, se canalizaron a otras instituciones.

Con aquellos adolescentes que fueron incluidos en el PIBA, entre 2010 y 2014, se realizó un seguimiento y una entrevista a un colateral. En total se realizaron 60 seguimientos completos a adolescentes junto con su respectivo colateral; cinco fueron hechos de manera presencial y 55 vía telefónica.

30 adolescentes concluyeron la intervención breve y fueron dados de alta; los otros 30 no lograron finalizar la intervención, pero completaron la evaluación inicial y acudieron, por lo menos, a la primera sesión (balance decisional y establecimiento de meta). Una vez que los usuarios acudieron a su cita programada o accedieron a realizar la entrevista de seguimiento, firmaron el consentimiento informado o se les leyó vía telefónica. Se informó a cada participante que los datos del seguimiento son confidenciales y que serían utilizados con fines de inves- tigación; se les comentó, asimismo, que el propósito del seguimiento es apoyar a los usuarios en el proceso de cambio posterior al tratamiento.

Después de que los usuarios aceptaron participar, se procedió a aplicar la entrevista de seguimiento completa y el cuestionario de confianza situacional; finalmente, se pidió que un acompañante o colateral mayor de edad corroborara la información proporcionada por el adolescente en la entrevista.

Al concluir la entrevista, se aclararon dudas y se escucharon sugerencias del usuario y el colateral. En caso de ausencia de recaídas y si el usuario no necesitaba sesiones adicionales, se reforzaba el mantenimiento de su meta de abstinencia o reducción, se le daban las gracias por su participación y se le proporcionaban teléfonos de contacto del Centro. En el caso de detectar caídas o recaídas, se consideraba la pertinencia de retomar algunas sesiones del PIBA, se refería al participante a otra institución o se le proporcionaba consejería breve para recordar las metas y estrategias de cambio. La duración de cada seguimiento fue de una hora en promedio. Ya obtenida la información de cada usuario y su colateral, se procedió a la captura de la información para analizarla y obtener resultados; se hicieron comparaciones de los datos recabados durante la evaluación inicial y los datos obtenidos en el seguimiento (Rodríguez, 2016).

\section{Análisis estadístico}

Las variables cantidad y frecuencia de consumo se midieron en escala ordinal. Se realizó un análisis de medidas repetidas para comparar el antes y el después de la intervención, se eligió la Prueba de los Rangos con Signo Wilcoxon.

La variable consecuencias negativas asociadas con el consumo fue medida en un nivel de escala nominal. Se realizó un análisis de medidas repetidas con la Prueba Mcnemar de muestras relacionadas (dicotómica) para comparar el antes y el después de la intervención.

La variable autoeficacia fue medida en una escala de intervalo. Se realizó un análisis de medidas repetidas para comparar el antes y el después de la intervención y se eligió la Prueba $t$ de Student para muestras relacionadas.

\section{RESULTADOS}

\section{A. Cantidad de consumo de las tres sustancias (al- cohol, mariguana e inhalables) en los usuarios que asistieron al PIBA}

La Tabla 4 compara el porcentaje de consumo de alcohol por ocasión preintervención vs. seguimiento, en ella se aprecia que de los 13 consumidores de alcohol, 69.2\% 
Tabla 4

Comparación de la variable cantidad de consumo de alcohol

\begin{tabular}{|c|c|c|c|c|c|c|}
\hline & \multicolumn{2}{|c|}{$\begin{array}{l}\text { Grupo alcohol } \\
\quad(n=13)\end{array}$} & \multicolumn{2}{|c|}{$\begin{array}{l}\text { Grupo alcohol alta } \\
(n=6)\end{array}$} & \multicolumn{2}{|c|}{$\begin{array}{l}\text { Grupo alcohol baja } \\
\qquad(n=7)\end{array}$} \\
\hline & Preintervención & Seguimiento & Preintervención & Seguimiento & Preintervención & Seguimiento \\
\hline \multicolumn{7}{|l|}{ Cantidad consumida } \\
\hline Abstemios & $0 \%$ & $46.2 \%$ & $0 \%$ & $33.3 \%$ & $0 \%$ & $57 \%$ \\
\hline De 1 a $4 U_{B S}{ }^{1}$ & $30.8 \%$ & $23 \%$ & $16.7 \%$ & $50 \%$ & $43 \%$ & $0 \%$ \\
\hline De 5 a 9 UBS & $53.8 \%$ & $30.8 \%$ & $66.6 \%$ & $16.7 \%$ & $43 \%$ & $43 \%$ \\
\hline 10 o más UBS & $15.4 \%$ & $0 \%$ & $16.7 \%$ & $0 \%$ & $14 \%$ & $0 \%$ \\
\hline Prueba Wilcoxon & $\begin{array}{l}Z=-2.511 \\
\text { Sig. asintótica }\end{array}$ & (bilateral) .012 & $\begin{array}{l}Z=-2.070 \\
\text { Sig. asintótica }\end{array}$ & (bilateral) .038 & $\begin{array}{l}Z=-1.511 \\
\text { Sig. asintótica }\end{array}$ & bilateral).131 \\
\hline
\end{tabular}

Nota: ${ }^{1}$ Unidad de Bebida Estándar.

Tabla 5

Comparación de la variable cantidad de consumo de mariguana

\begin{tabular}{|c|c|c|c|c|c|c|}
\hline & \multicolumn{2}{|c|}{$\begin{array}{l}\text { Grupo mariguana } \\
\qquad(n=37)\end{array}$} & \multicolumn{2}{|c|}{$\begin{array}{c}\text { Grupo mariguana } \\
\text { alta } \\
(n=18)\end{array}$} & \multicolumn{2}{|c|}{$\begin{array}{c}\text { Grupo mariguana } \\
\text { baja } \\
(n=19)\end{array}$} \\
\hline & Preintervención & Seguimiento & Preintervención & Seguimiento & Preintervención & Seguimiento \\
\hline \multicolumn{7}{|l|}{ Cantidad consumida } \\
\hline Abstemios & $0 \%$ & $51.4 \%$ & $0 \%$ & $72.2 \%$ & $0 \%$ & $31.6 \%$ \\
\hline Algunas fumadas & $10.8 \%$ & $13.5 \%$ & $16.7 \%$ & $16.6 \%$ & $5.3 \%$ & $10.5 \%$ \\
\hline Medio cigarro & $21.6 \%$ & $5.4 \%$ & $16.7 \%$ & $5.6 \%$ & $26.3 \%$ & $5.3 \%$ \\
\hline Un cigarro & $43.2 \%$ & $24.3 \%$ & $55.6 \%$ & $5.6 \%$ & $31.6 \%$ & $42.1 \%$ \\
\hline Dos cigarros & $13.5 \%$ & $5.4 \%$ & $5.6 \%$ & $0 \%$ & $21.1 \%$ & $10.5 \%$ \\
\hline Tres cigarros & $2.7 \%$ & $0 \%$ & $5.6 \%$ & $0 \%$ & $0 \%$ & $0 \%$ \\
\hline Más de tres cigarros & $8.1 \%$ & $0 \%$ & $0 \%$ & $0 \%$ & $15.8 \%$ & $0 \%$ \\
\hline Prueba Wilcoxon & \multicolumn{2}{|c|}{$\begin{array}{l}Z=-4.843 . \\
\text { Sig. asintótica (bilateral) } .000\end{array}$} & \multicolumn{2}{|c|}{$\begin{array}{l}Z=-3.677 . \\
\text { Sig. asintótica (bilateral) } .000\end{array}$} & \multicolumn{2}{|c|}{$\begin{array}{l}Z=-3.222 . \\
\text { Sig. asintótica (bilateral) } .001\end{array}$} \\
\hline
\end{tabular}

mantuvo un consumo sin riesgo. De los seis usuarios dados de alta, 83.3\% reportó abstinencia o un consumo de bajo riesgo ${ }^{1}$ en el seguimiento. De los siete usuarios con baja, 57\% reportó abstinencia. El análisis estadístico mostró diferencias significativas preintervención vs. seguimiento en el grupo total de usuarios con consumo de alcohol $(Z=-2.511)$ y en el grupo de usuarios con alta $(Z=-2.070)$, pero no en el grupo de baja $(Z=-1.511)$.

En los usuarios que consumieron mariguana se encontró lo siguiente: de los 18 usuarios del grupo de alta, $72.2 \%$ alcanzó la abstinencia; y de los 19 con baja, 31.6\% la logró. El análisis estadístico señaló que existen diferencias significativas preintervención vs. seguimiento en los tres grupos: total de usuarios $(Z=-4.843)$, usuarios con alta $(Z=-3.677)$ y usuarios de baja $(Z=-3.222)$, ver Tabla 5.

En la Tabla 6 se muestra que de los 10 participantes que reportaron consumo de inhalables, 60\% logró la abstinencia; en este grupo se observaron diferencias significativas preintervención vs. seguimiento $(Z=$ -2.414). En los seis casos del grupo de alta, 50\% de los participantes se mantuvo sin consumo en el seguimiento, sin embargo, no se encontraron diferencias significativas en la medición pre-post intervención ( $Z=-1.847)$. En el grupo baja, 75\% mantuvo la abstinencia; no obs-

\footnotetext{
${ }^{1}$ Se considera un consumo de bajo riesgo en adolescentes, menos de 4 unidades de bebida estándar por ocasión.
} 
Tabla 6

Comparación de la variable cantidad de consumo de inhalables

\begin{tabular}{|c|c|c|c|c|c|c|}
\hline & \multicolumn{2}{|c|}{$\begin{array}{l}\text { Grupo inhalables } \\
(n=10)\end{array}$} & \multicolumn{2}{|c|}{$\begin{array}{l}\text { Grupo inhalables alta } \\
\qquad(n=6)\end{array}$} & \multicolumn{2}{|c|}{$\begin{array}{l}\text { Grupo inhalables baja } \\
(n=4)\end{array}$} \\
\hline & Preintervención & Seguimiento & Preintervención & Seguimiento & Preintervención & Seguimiento \\
\hline \multicolumn{7}{|l|}{ Cantidad consumida } \\
\hline Abstemios & $0 \%$ & $60 \%$ & $0 \%$ & $50 \%$ & $0 \%$ & $75 \%$ \\
\hline De 1 a 4 monas & $30 \%$ & $10 \%$ & $16.7 \%$ & $16.7 \%$ & $50 \%$ & $0 \%$ \\
\hline De 5 a 9 monas & $30 \%$ & $0 \%$ & $33.3 \%$ & $0 \%$ & $25 \%$ & $0 \%$ \\
\hline 10 o más monas & $40 \%$ & $29 \%$ & $50 \%$ & $33.3 \%$ & $25 \%$ & $25 \%$ \\
\hline Prueba Wilcoxon & \multicolumn{2}{|c|}{$\begin{array}{l}Z=-2.414 . \\
\text { Sig. asintótica (bilateral) } .016\end{array}$} & \multicolumn{2}{|c|}{$\begin{array}{l}Z=-1.847 \\
\text { Sig. asintótica (bilateral) } .066\end{array}$} & \multicolumn{2}{|c|}{$\begin{array}{l}Z=-1.633 . \\
\text { Sig. asintótica (bilateral) } .102\end{array}$} \\
\hline
\end{tabular}

Tabla 7

Comparación de la variable frecuencia de consumo

\begin{tabular}{|c|c|c|c|c|c|c|}
\hline & \multicolumn{2}{|c|}{$(n=60)$} & \multicolumn{2}{|c|}{$\begin{array}{c}\text { Grupo alta } \\
(n=30)\end{array}$} & \multicolumn{2}{|c|}{$\begin{array}{c}\text { Grupo baja } \\
\quad(n=30)\end{array}$} \\
\hline & Preintervención & Seguimiento & Preintervención & Seguimiento & Preintervención & Seguimiento \\
\hline \multicolumn{7}{|l|}{ Frecuencia de consumo } \\
\hline Sin consumo & $0 \%$ & $51.7 \%$ & $0 \%$ & $60 \%$ & $0 \%$ & $43.3 \%$ \\
\hline Menos de una vez al mes & $6.7 \%$ & $11.7 \%$ & $10 \%$ & $10 \%$ & $3.3 \%$ & $13.3 \%$ \\
\hline Una vez al mes & $20 \%$ & $11.7 \%$ & $30 \%$ & $20 \%$ & $10 \%$ & $3.3 \%$ \\
\hline Una vez a la semana & $15 \%$ & $16.7 \%$ & $20 \%$ & $10 \%$ & $10 \%$ & $23.3 \%$ \\
\hline Dos a tres veces a la semana & $45 \%$ & $6.7 \%$ & $30 \%$ & $0 \%$ & $60 \%$ & $13.3 \%$ \\
\hline Diariamente & $13.3 \%$ & $1.7 \%$ & $10 \%$ & $0 \%$ & $16.7 \%$ & $3.3 \%$ \\
\hline Prueba Wilcoxon & $\begin{array}{l}Z=-6.299 \\
\text { Sig. asintótica }\end{array}$ & (bilateral) .000 & $\begin{array}{l}Z=-4.471 \\
\text { Sig. asintótica }\end{array}$ & (bilateral) .000 & $\begin{array}{l}Z=-4.451 \\
\text { Sig. asintótica }\end{array}$ & (bilateral) .000 \\
\hline
\end{tabular}

Tabla 8

Comparación de la variable consecuencias negativas asociadas con el consumo

\begin{tabular}{|c|c|c|c|c|c|c|c|c|}
\hline & \multicolumn{2}{|c|}{$\begin{array}{c}\text { Alta } \\
\text { preintervención }\end{array}$} & \multicolumn{2}{|c|}{$\begin{array}{c}\text { Alta } \\
\text { seguimiento }\end{array}$} & \multicolumn{2}{|c|}{$\begin{array}{c}\text { Baja } \\
\text { preintervención }\end{array}$} & \multicolumn{2}{|c|}{$\begin{array}{c}\text { Baja } \\
\text { seguimiento }\end{array}$} \\
\hline & $n$ & $\%$ & $n$ & $\%$ & $n$ & $\%$ & $n$ & $\%$ \\
\hline \multicolumn{9}{|l|}{ Problemas experimentados } \\
\hline Daño físico & 1 & $3.3 \%$ & 0 & $0 \%$ & 1 & $3.3 \%$ & 0 & $0 \%$ \\
\hline Daño cognitivo & 12 & $40 \%$ & 7 & $23.3 \%$ & 14 & $46.7 \%$ & 9 & $30 \%$ \\
\hline Daño afectivo & 12 & $40 \%$ & 5 & $16.7 \%$ & 11 & $36.7 \%$ & 2 & $6.7 \%$ \\
\hline Problemas interpersonales & 23 & $76.7 \%$ & 8 & $26.7 \%$ & 25 & $83.3 \%$ & 12 & $40 \%$ \\
\hline Agresión & 7 & $23.3 \%$ & 1 & $3.3 \%$ & 0 & $0 \%$ & 1 & $3.3 \%$ \\
\hline Problemas escolares & 16 & $53.3 \%$ & 4 & $13.3 \%$ & 18 & $60 \%$ & 6 & $20 \%$ \\
\hline Prueba Mcnemar (dicotómica) & \multicolumn{4}{|c|}{$\begin{array}{l}\text { Nivel de significancia }=.05 \\
\text { Sig. asintótica. }=.000\end{array}$} & \multicolumn{4}{|c|}{$\begin{array}{l}\text { Nivel de significancia }=.05 \\
\text { Sig. } \text { asintótica. }=.001\end{array}$} \\
\hline
\end{tabular}


tante, tampoco se registraron diferencias significativas en la medición pre-post intervención $(Z=-1.633)$.

\section{B. Frecuencia de consumo, en el total de usuarios, y en los grupos de alta y baja del PIBA}

La Tabla 7 muestra una disminución en la frecuencia de consumo, ya que 31 casos se mantuvieron en abstinencia por un periodo mayor o igual a seis meses, posterior a la intervención: 18 del grupo de alta y 13 del de baja. Se encontró, también, que al menos una vez al mes consumieron nueve adolescentes del grupo de alta y cinco del grupo de baja. Por último, en el grupo de alta, tres adolescentes reportaron seguir consumiendo una vez a la semana y ninguno diariamente; mientras que en el grupo de baja, siete dijeron seguir consumiendo una vez a la semana y cinco a diario.

El análisis estadístico mostró que existen diferencias significativas preintervención vs. seguimiento en los tres grupos: total de usuarios ( $Z=-6.843)$, usuarios con alta $(Z=-4.471)$ y usuarios de baja $(Z=-4.451)$.

\section{Consecuencias negativas asociadas con el consumo}

En la Tabla 8 se muestra que en el área "problemas interpersonales" hubo un decremento de $76.7 \%$ a $26.7 \%$ en el grupo alta, y de $83.3 \%$ a $40 \%$ en el de baja. En el área "problemas escolares" se presentó una disminución de 53.3\% a 13.3\% en el grupo alta, y de 60\% a $20 \%$ en el de baja. Los cambios fueron significativos tanto en el grupo de alta $(p<.05)$, como en el de baja $(p<.05)$.

\section{Autoeficacia}

La Tabla 9 muestra la autoeficacia percibida, medida en ocho situaciones de riesgo en preintervención vs. seguimiento. En el grupo de alta se observaron incrementos significativos mediante la prueba $t$ de Student (con un a establecido a .95, se obtuvo un valor menor a .05), en todas las área de riesgo. En el grupo de baja, dos situaciones de riesgo no fueron significativas ("probando autocontrol", con un nivel de a de .125, y "conflicto con otros", con un nivel de a de.119).

Las Figuras 1 y 2 muestran los cambios en los adolescentes que asistieron al PIBA, en el nivel de autoeficacia percibida ante situaciones de riesgo, durante la evaluación inicial y el seguimiento. En el grupo de alta, los valores de autoeficacia son más altos que en el de baja, independientemente de que ambos grupos muestran mayor autoeficacia.

\section{CONCLUSIONES}

Los resultados de este estudio mostraron que el PIBA, aplicado por profesionales de la salud en formación, fue una herramienta eficaz para prevenir el consumo de sustancias en este escenario clínico. Además, redujo problemas asociados con el consumo y aumentó la autoeficacia en jóvenes inmersos en esta problemática; tal como se reportó en previas investigaciones (Martínez, 2003; Martínez et al., 2008; Martínez et al., 2010).

Este trabajo logró evaluar el mantenimiento del cambio en 60 adolescentes que asistieron al PIBA en el CPAHAV, entre 2010 y 2014. Se llevó a cabo un seguimiento posterior a seis meses y, en algunos casos, hasta de dos

Tabla 9

Nivel de significancia pre-post intervención en la variable autoeficacia

\begin{tabular}{|c|c|c|c|c|}
\hline \multirow[b]{2}{*}{ Prueba $t$ de Student para muestras relacionadas } & \multicolumn{2}{|c|}{ Grupo alta } & \multicolumn{2}{|c|}{ Grupo baja } \\
\hline & $t^{1}$ & $\begin{array}{c}\text { Sig. } \\
\text { (bilateral) }\end{array}$ & $t^{1}$ & $\begin{array}{c}\text { Sig. } \\
\text { (bilateral) }\end{array}$ \\
\hline \multicolumn{5}{|l|}{ Nivel de autoeficacia preintervención versus seguimiento } \\
\hline Emociones desagradables (E.M.) & -3.728 & .001 & -3.612 & .001 \\
\hline Malestar físico (M.F.) & -2.367 & .025 & -2.242 & .033 \\
\hline Emociones agradables (E.A.) & -2.779 & .009 & -3.079 & .005 \\
\hline Probando autocontrol (P.A.) & -2.988 & .006 & -1.581 & .125 \\
\hline Necesidad física (N.F.) & -3.187 & .003 & -3.414 & .002 \\
\hline Conflictos con otros (C.O.) & -2.103 & .044 & -1.608 & .119 \\
\hline Presión social (P.S.) & -2.400 & .023 & -4.715 & .000 \\
\hline Momentos agradables (M.A.) & -2.424 & .022 & -2.704 & .011 \\
\hline
\end{tabular}

Nota: ${ }^{1}$ Prueba de dos colas con un a establecido al .95 


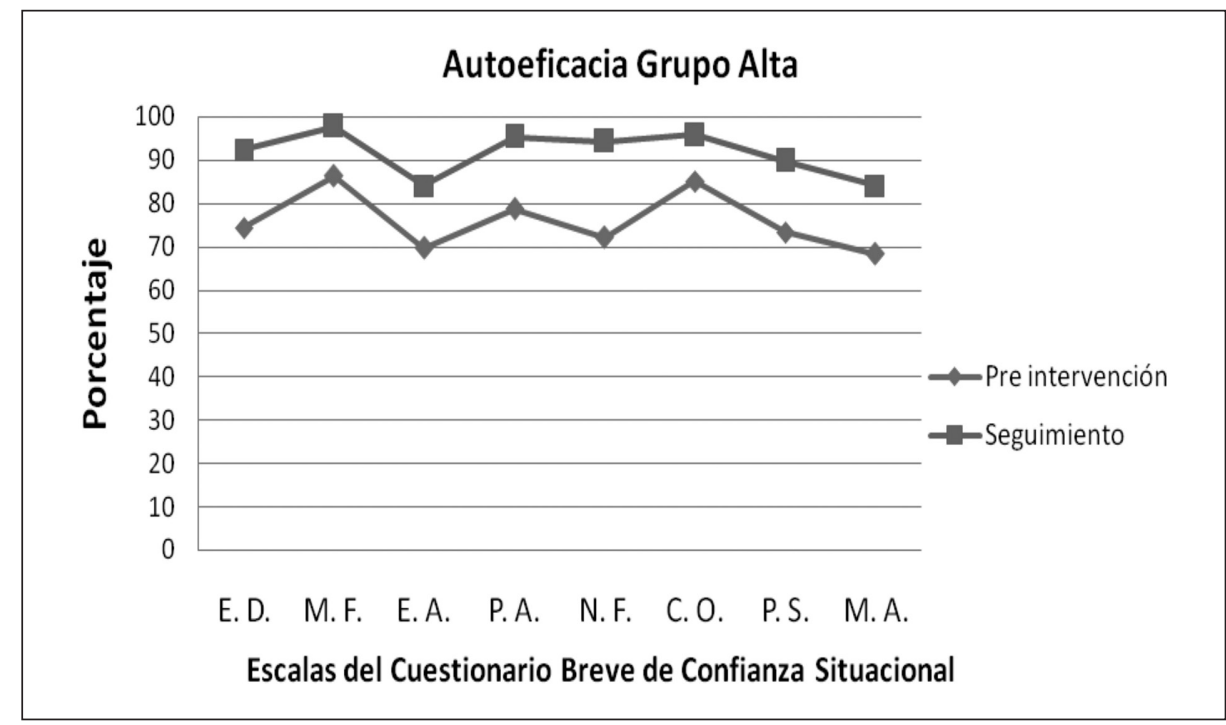

Figura 1. Autoeficacia grupo alta

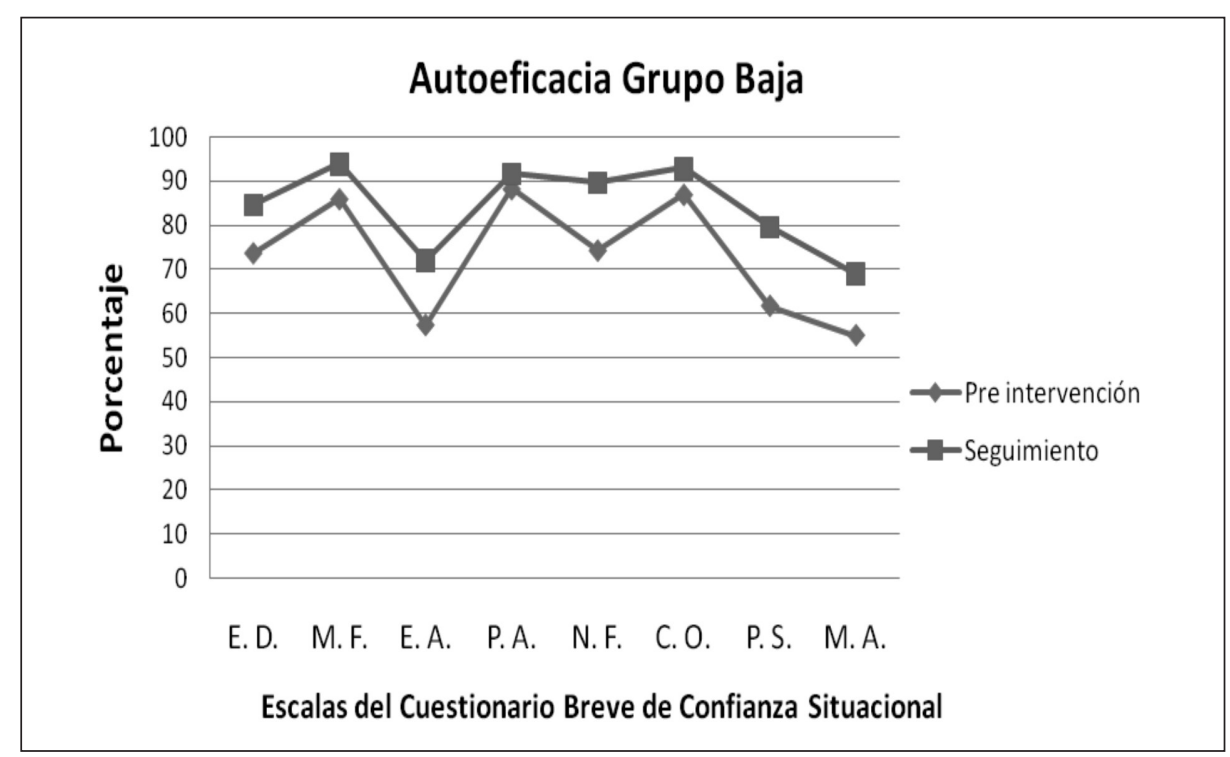

Figura 2. Autoeficacia grupo baja

años en el consumo de tres tipos de sustancias (alcohol, mariguana e inhalables). Al analizar los resultados, se determinó que los usuarios presentaron una reducción en las variables patrón de consumo y consecuencias negativas asociadas con el consumo; además, alcanzaron un incremento de la autoeficacia para rechazar el consumo. Aunado a esto, al examinar el desempeño posterior a la intervención, se observó que el grupo de usuarios que terminó el programa obtuvo mejores resultados, en contraste con quienes no lo concluyeron.
Aunque no todos los adolescentes lograron concluir la intervención breve, muchos de ellos lograron mantener resultados positivos, probablemente porque todos participaron en las siguientes etapas del PIBA: admisión, evaluación, inducción y la primera sesión de la fase de intervención, balance decisional. El balance decisional busca contemplar los pros y contras del consumo de sustancias, puede disminuir la ambivalencia y llevar a los jóvenes a decidir reducir o dejar el consumo de las sustancias. Dado lo anterior, sería plausible pensar que 
las fases previas al tratamiento, sumadas a una sesión de tratamiento del PIBA, ayudan a los jóvenes a tomar una decisión de cambio, sin necesidad de completar todo el programa.

Otro factor que pudo haber influido en la decisión de cambio de todos los participantes (que concluyeron o desertaron del PIBA) fue su obligación, al ser menores de edad, de informar a sus padres o tutores sobre su uso de sustancias psicoactivas. Al iniciarse en el consumo, los jóvenes, en general, tienen pocas consecuencias negativas; al encontrarse en etapas de precontemplación, no reconocen que tienen un problema con alguna conducta; sin embargo, ser identificados por los maestros y tener que acudir a un tratamiento los hace sensibilizarse y cambiar de etapa hacia la preparación del cambio.

La literatura científica nacional e internacional señala que la consejería breve, es decir una sola sesión de 30 minutos, puede motivar el cambio en etapas iniciales del consumo (Martínez et al., 2010; Tavera \& Martínez, 2008; Timko, Kong, Vittorio, \& Cucciare, 2016; Winters, Fahnhorst, Botzet, Lee, \& Lalone, 2012). Sin embargo, valdría la pena profundizar en esta hipótesis y determinar qué tanto una sola sesión bien estructurada puede lograr una transformación en los jóvenes que se inician en el uso de sustancias.

De igual manera, los resultados de este estudio corroboraron la eficacia de las intervenciones breves en la reducción de los patrones de consumo, el aumento de la autoeficacia percibida y la disminución de los problemas asociados con el consumo, como se reporta en otras investigaciones (Ayala, 2001; Ayala et al., 1998; Babor \& Grant, 1992; Espadero, 2008; Heather \& Kaner, 2001; Hewitt Ramírez \& Gantiva Díaz, 2009; Martínez, 2003; Sobell \& Sobell, 1996; Tiburcio et al., 2009). Estas intervenciones cumplen, además, con la demanda pública de reducir el costo social del abuso de drogas (Martínez \& Cabrera, 2011; Martínez et al., 2012; Tiburcio et al., 2009).

La efectividad de las intervenciones breves se mide con la reducción del uso de alcohol con patrones de riesgo en individuos con niveles bajos de dependencia (Ayala et al., 1998). El PIBA ha demostrado empíricamente ser costo-eficiente mediante la implementación de ensayos clínicos aleatorizados en adolescentes con problemas de consumo de drogas legales e ilegales, en poblaciones urbanas y rurales de nuestro país y por periodos mayores a seis meses (Martínez, 2003; Martínez et al., 2008).

Entre las limitaciones de este estudio se pueden mencionar que la muestra no fue aleatorizada y que los resultados obtenidos no fueron contrastados con un grupo control. Se propone que en futuras investigaciones se indague en variables como adherencia al tratamiento, nuevas modalidades tecnológicas de intervención breve y componentes eficaces en las intervenciones breves.

Por último, es importante insistir en la realización periódica y sistemática de los seguimientos a corto, mediano y largo plazo de las intervenciones breves, pues apoyan a los usuarios en su proceso y en el mantenimiento del cambio; además, es una actividad importante que promueve el desarrollo de nuevos conocimientos, otorga retroalimentación para los programas de tratamiento y contribuye en la formación de los profesionales de la salud que trabajan en el campo de la prevención.

\section{DECLARACIÓN DE LOS AUTORES}

La presente investigación no fue financiada por ninguna institución. Las opiniones, hallazgos y conclusiones aquí presentados son exclusivos de los autores.

\section{CONFLICTOS DE INTERÉS}

Los autores declaramos que no existe conflicto de intereses en cuanto al contenido del artículo ni con los datos presentados en el mismo.

\section{AGRADECIMIENTOS}

Al Centro de Prevención en Adicciones Dr. Héctor Ayala Velázquez (CPAHAV). Al Programa de Maestría y Doctorado de la UNAM. A la Maestría en Psicología de las Adicciones de la Facultad de Psicología de la UNAM.

\section{REFERENCIAS}

Annis, H. M., \& Davis, C. S. (1988). Self-efficacy and the prevention of alcoholic relapse: initial findings from a treatment trial. En: T. Baker \& D. Cannon (Eds.), Assessment and treatment of addictive disorders (pp. 88-112). Nueva York: Praeger.

Ayala, H. E. (2001). Desarrollo y evaluación de modelos de intervención para comportamientos adictivos en poblaciones urbanas y rurales. (Proyecto CONACYT G36266 H). México.

Ayala, H. E., Echeverría, L., Sobell, M. B., \& Sobell, L. C. (1998). Una alternativa de intervención breve y temprana para bebedores problema en México. Acta Comportamentalia: Revista Latina de Análisis del Comportamiento, 6(1), 71-96.

Babor, T. F., \& Grant, M. (Eds.). (1992). Project on identification and management of alcohol-related problems. Report on phase II: a randomized clinical trial of brief interventions in primary health care. Ginebra: Organización Mundial de la Salud.

Bandura, A. (1994). Self-efficacy. En V. S. Ramachaudran (Ed.), Encyclopedia of human behavior (pp. 71-81). Nueva York: Academic Press.

Bandura, A. (1986). Social foundations of thought and action: a social cognitive theory. Nueva Jersey: Prentice-Hall. 
Barragán, T. L., González, V. J., Medina-Mora, M. E., \& Ayala, V. H. (2005). Adaptación de un modelo de intervención cognoscitivo-conductual para usuarios dependientes de alcohol y otras drogas a población mexicana: un estudio piloto. Salud Mental, 28(1), 61-71

Echeverría, L., Ruiz, M., Salazar, L., Tiburcio, A., \& Ayala, V. H. (2004). Manual de detección temprana e intervención breve para bebedores problema. México: Facultad de Psicología, Universidad Nacional Autónoma de México.

Echeverría, S. L., \& Ayala, H. (1997). Cuestionario de confianza situacional: traducción y adaptación. México: Universidad Nacional Autónoma de México.

Espadero, D. B. (2008). Revisión sistemática sobre la efectividad de intervenciones breves en adolescentes consumidores de drogas, realizada según los distintos tipos de las mismas. Evidencias en Pediatría, 4(1), 23.

Heather, N., \& Kaner, E. (2001). Intervenciones breves: una oportunidad para reducir el consumo excesivo de alcohol entre los jóvenes. Adicciones, 13(4), 463-474.

Hervás, E. S., \& Tomás-Gradolli, V. (2002). Terapia breve en la adicción a drogas. Papeles del Psicólogo, 83, 49-54.

Hester, R. K., \& Miller, W. R. (1989). Handbook of alcoholism treatment approaches: effective alternatives. Nueva York: Pergamon Press.

Hewitt Ramírez, N., \& Gantiva Díaz, C. A. (2009). La terapia breve: una alternativa de intervención psicológica efectiva. Avances en Psicología Latinoamericana, 27(1), 165-176.

Instituto Nacional de Psiquiatría Ramón de la Fuente Muñiz, Comisión Nacional Contra las Adicciones, Secretaría de Salud. (2015). Encuesta Nacional de Consumo de Drogas en Estudiantes 2014: Reporte de Drogas. México: INPRFM.

Martínez, K. I. (2003). Desarrollo y evaluación de un modelo de intervención breve para adolescentes que inician el consumo de alcohol y otras drogas. (Disertación doctoral no publicada). México: Universidad Nacional Autónoma de México.

Martínez, K. I., Ávila, O., Pacheco, A., \& Lira, J. (2011). Investigaciones longitudinales: su importancia en el estudio del mantenimiento del cambio en las adicciones. Enseñanza e Investigación en Psicología, 16(2), 357-386.

Martínez, K. I., \& Cabrera, F. J. (2011). Factores asociados al proceso de recaída en adolescentes consumidores de alcohol. International Journal of Developmental and Educational Psychology, 1(2), 215-224.
Martínez, K. I., Pedroza-Cabrera, F. J., Salazar-Garza, M. L., \& Vacio-Muro, M. (2010). Evaluación experimental de dos intervenciones breves para la reducción del consumo de alcohol de adolescentes. Revista Mexicana de Análisis de la Conducta, 36(3), 35-53.

Martínez, K., Salazar, M., Jiménez, A., Ruiz, G., \& Ayala, V. H. (2012). Programa de intervención breve para adolescentes que inician el consumo abusivo de alcohol y otras drogas. Manual del Terapeuta. México: Centro Nacional para la Prevención y el Control de las Adicciones.

Martínez, K. I., Salazar-Garza, M. L., Pedroza-Cabrera, F. J., Ruiz-Torres, G. M., \& Ayala-Velázquez, H. E. (2008). Resultados preliminares del programa de intervención breve para adolescentes que inician el consumo de alcohol y otras drogas. Salud Mental, 31(2), 119-127.

Mingote, J. C., \& Requena, M. (Eds.). (2008). El malestar de los jóvenes: contextos, raíces y experiencias. España: Díaz de Santos.

Peñaloza, P. J. (2010). La juventud mexicana. Una radiografía de su incertidumbre. México: Porrúa.

Rodríguez, C. (2016). Intervención Breve para Adolescentes que Inician el Consumo de Alcohol y otras Drogas "PIBA". Resultados de seguimiento. (Reporte de experiencia profesional de maestría no publicado). México: Universidad Nacional Autónoma de México.

Sobell, M. B., \& Sobell, L. C. (1996). Problem drinkers: Guided self-change treatment. Nueva York: Guilford Press.

Tavera, R. S., \& Martínez, R. M. J. (2008). Guía para promotores Nueva Vida en Consejo Breve: IDEAS. México: Comisión Nacional contra las Adicciones.

Tiburcio, M., Carreño, S., Martínez, K., Echeverría, L., \& Ruiz, E. (2009). Cómo reducir el consumo de sustancias con intervenciones breves. México: Instituto Nacional de Psiquiatría Ramón de la Fuente Muñiz.

Timko, C., Kong, C., Vittorio, L., \& Cucciare, M. A. (2016). Screening and brief intervention for unhealthy substance use in patients with chronic medical conditions: a systematic review. Journal of Clinical Nursing, 25(21-22), 3131-3143. doi:10.1111/jocn.13244

Winters, K. C., Fahnhorst, T., Botzet, A., Lee, S., \& Lalone, B. (2012). Brief intervention for drug-abusing adolescents in a school setting: outcomes and mediating factors. Journal of Substance Abuse Treatment, 42(3), 279-288. doi: 10.1016/j.jsat.2011.08.005 